\title{
Relativistic Calculations of Atomic Structure
}

\section{B. Fricke}

Physics Departement, University of Kassel, D 35 Kassel, West Germany

Received September 2, 1983; accepted September 21, 1983
To, demonstrate the difference of these two descriptions Fig. 1 shows the electron density $\left(4 \pi r^{2}|\psi(r)|^{2}\right)$ of the $1 s, 2 s$ and $2 p$ states [2] in a one-electron mercury atom. The strong contraction towards smaller $r$ for the relativistic wavefunctions is especially pronounced for all $s$ and $p_{1 / 2}$ wavefunctions. This is called the Direct Relativistic Effect. Also the splitting of the non-relativistic $2 p$ wavefunction into the $2 p_{1 / 2}$ and $2 p_{3 / 2}$ relativistic wavefunctions can clearly be seen in Fig. 1(c).

This paper is intended to give a review on relativistic atomic structure calculations [1] with emphasis on the Multiconfigurational-Dirac-Fock method. Dr Crossley has summarized in his preceeding paper most of the non-relativistic methods which lead to good binding energies and transition probabilities. Both quantities are especially important in astrophysics and plasma physics. Since relativistic effects become increasingly important with increasing $Z$, I would like to concentrate first on a discussion of the differences between non-relativistic and relativistic calculations which can be best illustrated in the oneelectron case.

To describe the many electron atomic system the ansatz for the wavefunction and the resulting relativistic Multiconfiguration-Dirac-Fock (MCDF) procedure is discussed as a second point. In the last part the problems of this method its defficiencies as well as the additional contributions which go beyond the MCDF picture are discussed.

\section{Comparison of the non-relativistic and relativistic one-electron atom}

In the following the equations and quantities which describe a one-electron atom are listed.

Non-relativistic

Schrödinger equation.

The radial equation

$-\frac{1}{2} P^{\prime \prime}+\frac{l(l+1)}{2 r^{2}} P-\frac{z}{r} P=\epsilon P$

is one differential equation of second order.

There exists one radial function $P(r)$. The radial electron density $|P(r)|^{2}$ has $n-l-1$ zeros. The angular electron density has $l$ zeros.

\section{Hartree-(Dirac-) Fock description of many electron atoms}

The general equation which has to be solved in the case of a many-electron atom is the Schrödinger and Dirac equation, respectively where the electron-electron interaction is introduced in the Hamiltonian. The theoretical fundamental problems which are connected with this procedure in the case of the Dirac equation are discussed in general by Sucher [3].

If a 1-Slater-determinant is used as the ansatz for the wavefunction the variation of the total energy with respect to the radial functions leads to the Hartree-Fock-equations in the nonrelativistic case [4] and the Dirac-Fock-equations in the relativistic case [5]. The comparison of results of such calculations shows two features. First, the direct relativistic effect, i.e., the contraction of the $s$ and $p_{1 / 2}$ wavefunctions is very similar as discussed in the one-electron case. Second, the so called Indirect Relativistic Effect appears. Due to the strong contraction of the innermost wavefunctions the outer wavefunctions with large angular momenta become more shielded than in the non-relativistic case and expand. Both effects can very well be seen in Fig. 2 where the quantity $\langle r\rangle_{\text {rel }} /\langle r\rangle_{\text {non-rel }}$ is given as

Relativistic

Dirac equation.

The radial equation

$P^{\prime}+\frac{\kappa}{r} P-\left(2 c+\frac{1}{c}\left(\epsilon+\frac{z}{r}\right)\right) Q=0$

$Q^{\prime}+\quad Q^{\prime}\left(-\frac{\kappa}{r} \frac{z}{r}\right) P=0$

consists of two coupled differential equations of first order.

There exist two radial functions $P(r)$ and $Q(r)$ which are called the large and the small component respectively. The radia electron density $|P(r)|^{2}+|Q(r)|^{2}$ has no exact zeros. Also the angular electron density has no exact zeros.

Spectrum:

$E>m c^{2}$ continuum

$m c^{2}>E>-m c^{2}$ bound states

$E<-m c^{2}$ negative continuum

Good quantum numbers: $n, j, m_{j}$, Parity.

For $l>0$ there are two states instead:

$j_{1}=1+1 / 2 \quad$ The energy difference between these

$j_{2}=1-1 / 2 \quad$ states is the spin-orbit splitting. 
a)

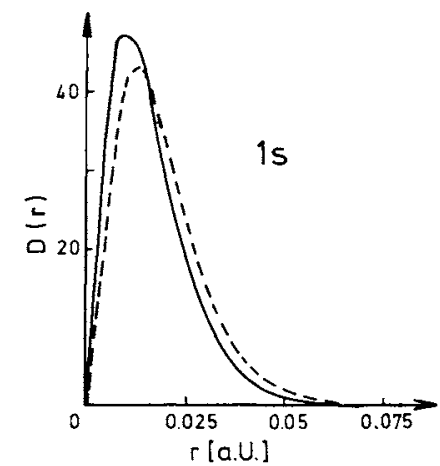

b)

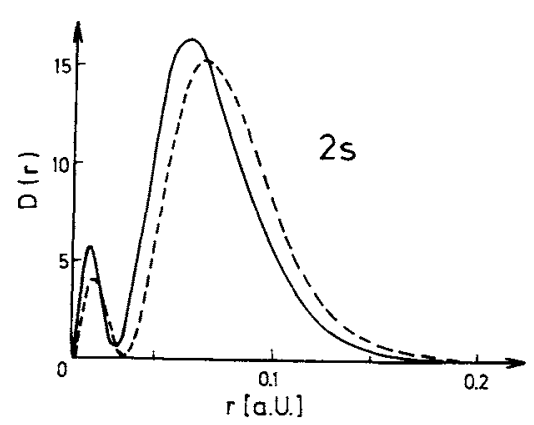

c)

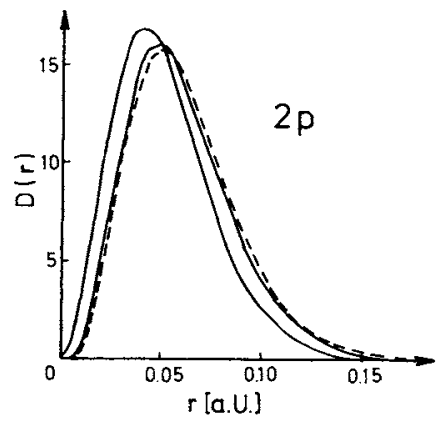

Fig. 1. Charge density $D=4 \pi r^{2}\left(|P|^{2}+|Q|^{2}\right)$ for an one-electron mercury atom. In (a) the $1 s$ in (b) the $2 s$ and in (c) the $2 p$ electron densities are given. — is the relativistic and -..- the non-relativistic calculation.

function of the main quantum number in fermium. The outermost $5 f$ wavefunctions expand due to the indirect relativistic effect whereas all $s$ wavefunctions contract, including those with large main quantum numbers although they are only loosely bound.

Since 1-Slater-determinental wavefunctions in general have no good angular momentum one has to construct so called Configuration State Functions (CSF) which are a linear combination of Slater determinants. These CSF have $J^{2}, M_{J}$ and the Parity as good quantum numbers in the relativistic case. If a linear combination of such CSF's are used as the ansatz for the wavefunctions to solve the Dirac equation one finally ends up with the Multiconfiguration-Dirac-Fock Method (MCDF) [6].

\section{Remarks on the MCDF-procedure}

The equation which has to be solved is

$H|\psi\rangle=E|\psi\rangle$

The ansatz for the total wavefunction $|\psi\rangle$ is a superposition of CSF

Fermium $(Z=100)$

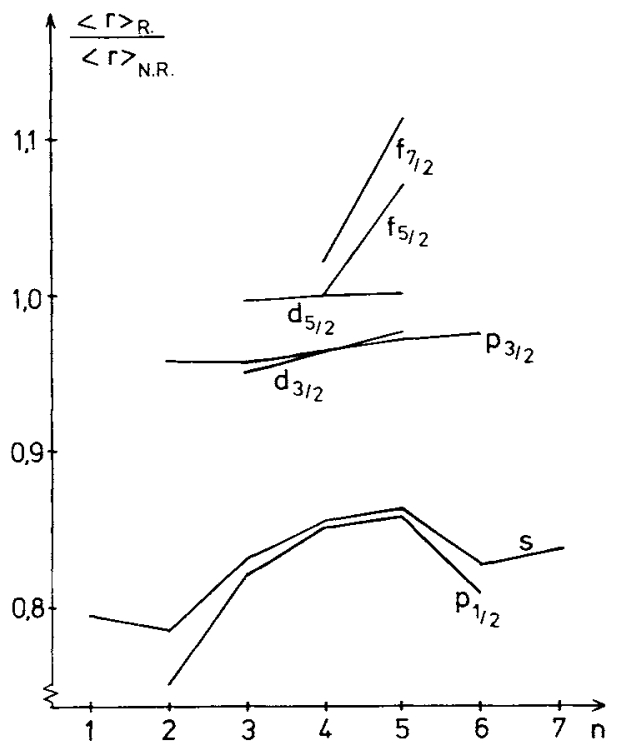

Fig. 2. The quotient of the expectation value of $r$ in a relativistic and a non-relativistic Hartree-Fock calculation of a fermium atom is shown as function of the main quantum number $n$.
$|\psi\rangle=\sum_{i=1}^{M} c_{i}\left|\phi_{i}\right\rangle$

The $\left|\phi_{i}\right\rangle$ are linear combinations of Slater determinants $\left|\alpha_{j}\right\rangle$

$\left|\phi_{i}\right\rangle=\sum_{j} \beta_{i j}\left|\alpha_{j}\right\rangle$

The Slater determinants $\left|\alpha_{j}\right\rangle$ are constructed from $N$ one-particle wavefunctions $\varphi$ which in the relativistic case are spinors of rank 4

$\varphi=\frac{1}{r}\left(\begin{array}{cc}P_{n k}(r) & \chi_{k}^{m_{j}} \\ i Q_{n k}(r) & \chi_{-k}^{m_{j}}\end{array}\right)$

$P(r)$ and $Q(r)$ are the large and small components of the radical functions. The angular part is a linear combination of spherical harmonics $Y_{l}^{m}$ and the spin function $S$ which is a spinor of rank 2

$\chi_{k}^{m_{j}}=\sum_{\sigma} Y_{l}^{m-\sigma} S^{\sigma}\left\langle l m-\sigma \frac{1}{2} \sigma \mid l \frac{1}{2} j m\right\rangle$

$k$ is the Dirac quantum number:

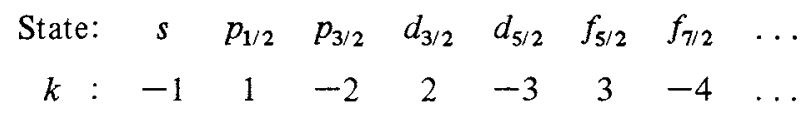

If we use the ansatz (3) for the wavefunctions which can be rewritten in the form

$|\psi\rangle=\sum_{i=1}^{M} c_{i}\left|\phi_{i}\right\rangle=\mathrm{c}^{t}|\varphi\rangle$

with

$\mathrm{c}^{t}=\left(c_{1}, c_{2}, \ldots, c_{M}\right) \quad$ and $\quad|\varphi\rangle=\left(\begin{array}{c}\left|\phi_{1}\right\rangle \\ \dot{\left.\phi_{M}\right\rangle}\end{array}\right)$

the total energy of a time-independent quantum mechanical system is then

$E=\langle\psi|H| \psi\rangle=\mathrm{c}^{t}\langle\varphi|H| \uparrow\rangle^{t} \mathbf{c}=\mathbf{c}^{t} H c$

with $(H)_{i j}=\left\langle\phi_{i}|H| \phi_{j}\right\rangle$.

The norm is given by

$1=\langle\psi \mid \psi\rangle=\mathrm{c}^{t} \mathrm{c}\left\langle\phi_{i} \mid \phi_{j}\right\rangle=\mathrm{c}^{t} \mathrm{c}=\sum_{i=1}^{M}\left|c_{i}\right|^{2}=1$.

In a Hartree-Fock-procedure the total energy $E$ is varied under the additional constraints that the norm of the total wavefunction is 1 and that the CSF's are orthogonal $\left\langle\phi_{i} \mid \phi_{j}\right\rangle=$ 
$\delta_{i j}$. In all relativistic versions which exist so far the authors $[7,8]$ use $\left\langle\varphi_{i} \mid \varphi_{j}\right\rangle=\delta_{i j}$ so that all Slater determinants are constructed from the same orthogonal set of atomic wavefunctions. This leads to the energy functional

$$
E=\mathrm{c}^{t} H \mathrm{c}-\omega\left(\mathrm{c}^{t} \mathrm{c}-1\right)+\sum_{i<j} \epsilon_{i j}\left(\left\langle\varphi_{i} \mid \varphi_{j}\right\rangle-1\right)
$$

where $\omega$ and $\epsilon_{i j}$ are Lagrange multipliers, which have to be varied (i) with respect to the expansion coefficients $c_{i}$ and (ii) with respect to the radial one-particle wavefunctions $P$ and $Q$.

The variation of (i) leads to the matrix equation

$H c=\omega c$

where $\omega$ is the total energy since $E=c^{t} H c=c^{t} \omega c=\omega$.

The variation of (ii) leads to the inhomogeneous coupled MCDF equations for the radial functions $P(r)$ and $Q(r)$

$P_{i}^{\prime}+\frac{k_{i}}{r} P_{i}=\left[2 c+\frac{1}{c}\left(\epsilon_{i}-V_{i}\right)\right] Q_{i}+X_{Q}$

$Q_{i}^{\prime}-\frac{k_{i}}{r} Q_{i}=-\frac{1}{c}\left(\epsilon_{i}-V_{i}\right) P_{i}+X_{p}$

$$
i=1,2, \ldots, N
$$

which are explained in detail in [6].

Since this variation is performed with respect to the radial part of the wavefunctions only this is a restricted Dirac-Fockprocedure.

In a practical calculation one starts with good guesses of the potential $V_{i}$ and the expansion coefficients $c_{i}$, calculates eq. (11) to get new $P_{i}$ and $Q_{i}$ then calculates the matrix $H$ and diagonalizes eq. (10) which in turn allows a new calculation of eq. (11), etc. This has to be done until convergence is achieved both for the $P$ 's and $Q$ 's as well as the $c_{i}$. The two programs which solve this problem are the programs by Desclaux [7] and Grant et al. [8]. A large number of such calculations have since been performed by the authors as well as a large number of other people [9].

To give a very simple example of the usefulness of these programs I discuss the configuration $s p$ in the isoelectronic series of $\mathrm{Be}$. The pure $j j$-configurations are: $s p_{1 / 2}$ with $J=0,1$ and $s p_{3 / 2}$ with $J=1,2$. Thus the two $J=1$ states can be described in the $L S$ picture by

$\left|{ }^{3} P_{1}\right\rangle=\sqrt{2 / 3}\left|s p_{1 / 2}\right\rangle+\sqrt{1 / 3}\left|s p_{3 / 2}\right\rangle$

$\left.{ }^{1} P_{1}\right\rangle=-\sqrt{1 / 3}\left|s p_{1 / 2}\right\rangle+\sqrt{2 / 3}\left|s p_{3 / 2}\right\rangle$

Of course in reality one has neither pure $L S$ coupling nor pure $j j$-coupling but intermediate coupling so that the ${ }^{1} P_{1}$ state for example can be written as

$\left.{ }^{1} P_{1}\right\rangle=-a\left|s p_{1 / 2}\right\rangle+b\left|s p_{3 / 2}\right\rangle$

in the presence of additional $1 s^{2}$ electrons.

A MCDF calculation of this system not only yields the one particle wavefunctions but also the coefficients $a$ and $b$. Figure 3 shows the results of such calculations for the factor $a$ which clearly shows the quick breakdown from pure $L S$ coupling at very small $Z$ to nearly pure $j j$-coupling for very large $Z$ for the isoelectronic series of $\mathrm{Be}$.

\section{Approximations and problems in the MCDF calculations}

The Multiconfigurational-Dirac-Fock procedure would be an

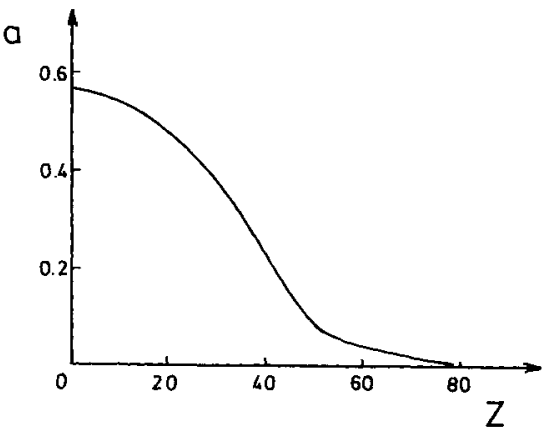

Fig. 3. Expansion coefficient $a$ for the intermediate wavefunction of the ${ }^{1} P_{1}$ state in the isoelectronic Be-series. The wavefunction is defined as $\left.{ }^{1} P_{1}\right\rangle=-a\left|s p_{1 / 2}\right\rangle+b\left|s p_{3 / 2}\right\rangle$.

exact solution for the given Hamiltonian if the number of configurations would be infinite. Since this never can be achieved the result always is an approximation!

In general one can say that the calculations become better with increasing number of configurations, but since a good intermediate coupling always needs all possible $j i$-configuration state functions at least all CSF's, which are possible within the same shell of the atomic one-particle functions, should be taken into account.

As stated above it is necessary in the MCDF procedure that all CSF are orthogonal to each other. This means that the oneparticle atomic wave-functions from which the Slater determinants are constructed are orthogonal to each other only within one CSF. If one would allow for many different sets of one-particle atomic wavefunctions the MCDF programs would become very much more difficult. That is the reason why up to now only one set of one-particle orthogonal atomic wavefunctions $\varphi$ are used to construct all CSF's. The error introduced by this procedure usually is expected to be small but this should be proven in each case [10]. Again an infinite number of atomic basis states would solve this problem too.

The finite, and different, number of CSF for each calculation of different total angular momentum $J$ introduces an additional error which first was discussed in detail by Huang et al. [11]. The best example for this deficiency of the MCDF method now in use is the fine-structure splitting between the ${ }^{2} P_{1 / 2}$ and ${ }^{2} P_{3 / 2}$ states in B-like ions. In a single-configuration calculation this is the splitting between the $\left(2 s^{2} 2 p_{1 / 2}\right)^{2} P_{1 / 2}$ and $\left(2 s^{2} 2 p_{3 / 2}\right)^{2} P_{3 / 2}$ state. If one does a MCDF calculation taking into account all CSF constructed from all atomic functions with $n=2$ one gets the two states (plus the $1 s^{2}$-core)

$$
\begin{aligned}
|J=1 / 2\rangle= & a\left|2 s^{2} 2 p_{1 / 2}\right\rangle+b\left|2 p_{1 / 2} 2 p_{3 / 2}^{2}\right\rangle \\
|J=3 / 2\rangle= & a\left|2 s^{2} 2 p_{3 / 2}\right\rangle+b\left|2 p_{1 / 2}^{2} 2 p_{3 / 2}\right\rangle+c\left|2 p_{3 / 2}^{3}\right\rangle \\
& +d\left|2 p_{1 / 2} 2 p_{3 / 2}^{2}\right\rangle
\end{aligned}
$$

Table I. Spin-orbit splitting (in $\mathrm{cm}^{-1}$ ) in the ground state of $B$ like ions

\begin{tabular}{lcclrc}
\hline Ion & SC & MCDF & $\begin{array}{l}\text { MCDF } \\
(\alpha=0)\end{array}$ & Corrected & Exp* \\
\hline $\mathrm{B}$ & 15.7 & 435.3 & 419.6 & 15.7 & 16 \\
$\mathrm{C}^{+}$ & 64.4 & 271.5 & 208.8 & 62.7 & 63.42 \\
$\mathrm{~N}^{2+}$ & 179 & 335.8 & 163.4 & 172.4 & 174.5 \\
$\mathrm{Ne}^{5+}$ & 1346 & 1472 & 174 & 1298 & 1310 \\
$\mathrm{Si}^{9+}$ & 7194 & 7183 & 215 & 6968 & 6990 \\
$\mathrm{Ar}^{13+}$ & 23286 & 22856 & 244 & 22612 & 22655.9 \\
\hline
\end{tabular}

* For references see [11]. 
Table II. (a) Fine structure interval of $1 s 2 s 2 p^{4} P^{0}$ and $1 s 2 p^{2}{ }^{4} P^{\mathrm{e}}$ term (in $\mathrm{cm}^{-1}$ ) in Li-like $F$ and Mg. (b) Wavelength in $\AA$ for ${ }^{4} P_{J}^{0}-{ }^{4} P_{J}^{\mathrm{e}}$ transitions for Li-like $F$ and $M g$

\begin{tabular}{|c|c|c|c|c|c|c|c|}
\hline \multirow[t]{2}{*}{$Z$} & & \multicolumn{3}{|l|}{${ }^{4} P^{0}$} & \multicolumn{3}{|l|}{${ }^{4} P^{\mathrm{e}}$} \\
\hline & & $5 / 2-3 / 2$ & \multicolumn{2}{|c|}{$3 / 2-1 / 2$} & \multicolumn{2}{|l|}{$5 / 2-3 / 2$} & $3 / 2-1 / 2$ \\
\hline 9 exp. $[14 a]$ & & 713 & \multicolumn{2}{|c|}{211} & \multicolumn{2}{|l|}{474} & 503 \\
\hline MCDF-EAL [12] & & 718.8 & \multicolumn{2}{|c|}{219.5} & \multicolumn{2}{|l|}{475.37} & 504.15 \\
\hline $\mathrm{MCDF}-\mathrm{OL}[13]$ & & 706 & \multicolumn{2}{|c|}{201} & \multicolumn{2}{|l|}{456} & 505 \\
\hline 12 exp. [14b] & & $2708(20)$ & \multicolumn{2}{|c|}{$1010(20)$} & \multicolumn{2}{|l|}{$1995(25)$} & $1824(35)$ \\
\hline MCDF-EAL [12] & & 2731.3 & \multicolumn{2}{|c|}{1038.6} & \multicolumn{2}{|l|}{2018.1} & 1835.7 \\
\hline MCDF-OL [13] & & 2742 & \multicolumn{2}{|c|}{968} & \multicolumn{2}{|l|}{2016} & 1855 \\
\hline \multirow[t]{2}{*}{$Z$} & \multicolumn{7}{|l|}{$J-J^{\prime}$} \\
\hline & $3 / 2-5 / 2$ & $1 / 2-3 / 2$ & $3 / 2-3 / 2$ & $5 / 2-5 / 2$ & $1 / 2-1 / 2$ & $3 / 2-1 / 2$ & $5 / 2-3 / 2$ \\
\hline $9 \exp .[14 a]$ & 818.09 & 819.89 & 821.37 & 822.91 & 823.42 & 824.99 & 826.23 \\
\hline MCDF-EAL [12] & 817.1 & 818.8 & 820.3 & 822.0 & 822.2 & 823.7 & 825.2 \\
\hline MCDF-OL $[13]$ & 810.3 & 811.9 & 813.6 & 815.1 & 815.2 & 816.7 & 818.4 \\
\hline 12 exp. [14b] & 577.62 & 580.93 & 584.35 & 586.80 & 587.10 & 590.70 & 593.75 \\
\hline MCDF-EAL [12] & 577.00 & 580.27 & 583.79 & 586.23 & 586.52 & 590.12 & 593.25 \\
\hline MCDF-OL [13] & 573.72 & 577.12 & 580.44 & 582.89 & 583.44 & 596.75 & 589.82 \\
\hline
\end{tabular}

MCDF calculations for these two states lead to an energy splitting which is by far too large as can be seen in row 3 of Table I which is taken from [11]. The reason for this deficiency is the inequivalent treatment of the correlation problem for the two $J$-states. (For details see [11]). One possible cure is to perform the same calculations with the fine structure constant $\alpha=0$ (or $c \rightarrow \infty$ ) which is a non-relativistic analogue calculation. Since the correlation is treated inequivalent one also gets a splitting in this case (see row 4 of Table I) although nonrelativistically both levels should be degenerate. If one corrects row 3 with row 4 one gets the results of row 5 which indeed are better than the single-configuration calculations. From a general point of view this unphysical correlation energy also is a consequence of the finite number of CSF and associated finite number of atomic one-particle wavefunctions.

But how to overcome this difficulty in an acutal finite calculation? The proposal by Grant [8] is, to calculate the radial functions in eq. (11) from an energy functional of the form

$E=$ const. $\sum_{i}(2 J+1) E_{i}$

where the sum goes over all possible $i j$-coupled CSF belonging to the set of atomic one-particle wavefunctions which are taken into account. If one then uses these radial functions to determine the energy matrix $\boldsymbol{H}$ only one diagonalization is required to get all configuration energies as well as expansion coefficients $c_{i}$. This method is called MCDF-EAL (EAL for extended average level). Since this method does not need a selfconsistent procedure it is very cheap. The general method described above needs a full selfconsistent treatment for both the $P$ 's and $Q$ 's as well as the $c_{i}$ for each state calculated. This method therefore is called MCDF-OL (OL for optimal level). An example and comparison for both methods $[12,13]$ is given in Table II, where the calculation of the fine-structure within the ${ }^{4} P^{\mathbf{e}}$ and ${ }^{4} P^{0}$ states as well as the transition energies between them in Li-like systems is given. Indeed Table II shows that the MCDFEAL method is nearer to the experimental [14] results although the absolute difference between the calculations and the exper- iment is nearly constant in both cases. The effect of the averaging procedure of eq. (12) probably is just the constant difference between the two calculations.

This short description probably made clear that there are computer programs available which enable everyone to calculate binding energies, transition energies and wavefunctions with this sophistication discussed above. Examples of calculations of transition probabilities with this method are given in [15].

\section{Additional corrections}

In the results discussed in Table II additional contributions which go beyond the usual MCDF procedure are already included within some approximation. These corrections are corrections due to a more realistic interaction between the charged particles within the atom.

The main contribution comes from the Breit-operator which is an expression for the transverse part of the $e-e$ interaction. This is often called magnetic interaction and retardation. In the usually used approximate form the operator reads

$H_{\mathrm{Br}}=-e^{2}\left(\frac{\boldsymbol{\alpha}_{i} \cdot \boldsymbol{\alpha}_{j}}{r_{i j}}+\frac{\left(\boldsymbol{\alpha}_{i} \nabla_{i}\right)\left(\boldsymbol{\alpha}_{j} \nabla_{j}\right) \mathbf{r}_{i j}}{2}\right)$

Details can be found in [16-20]. This operator normally is calculated in perturbation theory to correct for the total energy but is usually not included in the self-consistent process to correct for the wavefunctions. On the other hand the contribution due to this operator can now be calculated in a coupling to good $J$ [18-20].

The second largest correction is due to vacuum fluctuation or self-energy. Usually Mohr's one-electron QED values [21, 22] are used in a somehow screened way [19]. This procedure is the best one can use at present but has no sound theoretical justification. Better many-electron QED calculations are urgently needed.

The third correction is the vacuum polarisation which is a correction of the nucleus-electron interaction. The main part of it is the long known Uehling potential [23]. 
Table III. Contribution of the Breit-operator and the QED corrections for the innermost electron levels in Th and Fm

\begin{tabular}{llrr}
\hline & & Thorium & Fermium \\
\hline (a) Vacuum polarisation & $1 s$ & $-80 \mathrm{eV}$ & $-148 \mathrm{eV}$ \\
Uehling term & $2 p_{1 / 2}$ & $-2 \mathrm{eV}$ & $-4 \mathrm{eV}$ \\
higher order & $1 s$ & $+4 \mathrm{eV}$ & $+8 \mathrm{eV}$ \\
(b) Vacuum fluctuation & $1 s$ & $+306 \mathrm{eV}$ & $+457 \mathrm{eV}$ \\
& $2 p_{1 / 2}$ & $+7 \mathrm{eV}$ & $+15 \mathrm{eV}$ \\
(c) Breit-term & & & \\
magnetic part & $1 s$ & $+492 \mathrm{eV}$ & $+715 \mathrm{eV}$ \\
& $2 p_{1 / 2}$ & $+100 \mathrm{eV}$ & $+153 \mathrm{eV}$ \\
retardation & $1 s$ & $-36 \mathrm{eV}$ & $-41 \mathrm{eV}$ \\
& $2 p_{1 / 2}$ & $-10 \mathrm{eV}$ & $-13 \mathrm{eV}$ \\
\hline
\end{tabular}

Table III shows the magnitude of these effects in very heavy atoms for which it is known since a long time that these contributions are absolutely necessary to get good agreement between theory and experiment $[16,17]$. That these additional contributions are also important in low $Z$ systems clearly is demonstrated in Table IV which show the newest high precission measurements and calculations in He-like iron [10].

Table IV. Contributions to the transition energies of the ${ }^{1} P$ and ${ }^{3} \mathrm{P}$ to ${ }^{1} \mathrm{~S}$ transitions in He-like iron (in eV)

\begin{tabular}{lrrr}
\hline & ${ }^{1} P_{1}-{ }^{1} S_{0}$ & \multicolumn{1}{c}{${ }^{3} P_{1}-{ }^{1} S_{0}$} & \multicolumn{1}{c}{${ }^{3} P_{2}-{ }^{1} S_{0}$} \\
\hline Hartree-Fock & 6638.81 & 6616.77 & 6616.77 \\
Dirac corr. & 70.22 & 59.00 & 74.06 \\
Breit int. & -6.10 & -5.70 & -6.08 \\
QED & -3.52 & -3.58 & -3.55 \\
Correlation & 1.29 & 1.30 & 1.28 \\
$\quad$ MCDF minus SCDF) & & & \\
Theory & 6700.70 & 6667.79 & 6682.48 \\
Experiment & 6701.9 & 6667.5 & 6682.7 \\
\hline
\end{tabular}

\section{References}

1. An excellent long write up of the foundation of relativistic theory as well as all methods and problems is given in: Relativistic Effects in Atoms, Molecules and Solids (Edited by G. L. Malli). Plenum Press, New York and London (1983).

2. Burke, V. M. and Grant, I. P., Proc. Phys. Soc. 90, 297 (1967).

3. Sucher, J., pp. 1-54 in [1] and references therein.

4. Froese-Fischer, C., The Hartree-Fock Method for Atoms. Wiley, New York (1977).

5. Grant, I. P., Adv. Phys. 19, 747 (1970).

6. Desclaux, J. P., pp. 115-143 and Grant, I. P., pp. 55-114 in [1].

7. Desclaux, J. P., Comp. Phys. Comm. 9, 31 (1975).

8. Grant, I. P., McKenzie, B. J., Norrington, P. H., Mayers, D. F. and Pyper, N. C., Comp. Phys. Comm. 21, 207 (1980).

9. See for example the references in the articles by Grant, I. P., Desclaux, J. P. and Pyper, N. C., in [1].

10. Briand, J. P., Tavernier, M., Marrus, R., Gould, H. and Desclaux, J. P., To be published in Phys. Rev. A.

11. Huang, K. N., Kim, Y. K., Cheng, K. T. and Dexclaux, J. P., Phys. Rev. Lett. 48, 1245 (1982).

12. Hatta, J. and Grant, I. P., J. Phys. B16, 915 (1983).

13. Rosén, A., Olsson, G. and Fricke, B., To be published.

14. Martinson, I., Denne, B., Ekberg, J. O., Engström, L., Huldt, S., Jupén, C., Litzén, U., Mannervik, S. and Trigueiros, A., Phys. Scripta 27, 201 (1983), as well as the experimental references in [12].

15. Desclaux, J. P. and Kim, Y. K., J. Phys. B8, 1177 (1975); Kim, Y. K. and Desclaux, J. P., Phys. Rev. Lett. 36, 139 (1976); Cheng, K. T., Kim, Y. K. and Desclaux, J. P., At. Data Nucl. Data Table 24, 111 (1979).

16. Mann, J. P. and Johnson, W. R., Phys. Rev. A3, 1267 (1971).

17. Fricke, B., Desclaux, J. P. and Waber, J. T., Phys. Rev. Lett. 28, 714 (1972).

18. Cheng, K. T., Desclaux, J. P. and Kim, Y. K., J. Phys. B11, L359 (1978).

19. Desclaux, J, P., in Proceedings of the Workshop on Foundations of the Relativistic Theory of Atomic Structure, ANL-Report ANL-80126 (1981).

20. McKenzie, B. J., Grant, I. P. and Norrington, P. H., Comp. Phys. Comm. 21, 233 (1980).

21. Mohr, P., in Proceedings of the Workshop on Foundations of the Relativistic Theory of Atomic Structure. ANL-Report, ANL-80-126 (1981).

22. Mohr, P., pp. 145-167 in [1].

23. Uehling, E. A., Phys. Rev. 48, 55 (1935). 